Article

\title{
Measurement of the Verdet Constant of Polarization-Maintaining Air-Core Photonic Bandgap Fiber
}

\author{
Ningfang Song, Xiaoyang Wang ${ }^{\mathbb{D}}$, Xiaobin $\mathrm{Xu}$ *, Wei Cai and Chunxiao Wu \\ Department of Opto-electronics Engineering, Beihang University, Beijing 100191, China; \\ Songnf@buaa.edu.cn (N.S.); xiaoyang_wang@buaa.edu.cn (X.W.); sdfz174caiwei@126.com (W.C.); \\ wuchunxiao34@126.com (C.W.) \\ * Correspondence: xuxiaobin@buaa.edu.cn; Tel.: +86-10-8231-6887
}

Received: 5 July 2017; Accepted: 15 August 2017; Published: 17 August 2017

\begin{abstract}
We propose a method based on the white-light interference technique for measuring the Verdet constant of a polarization-maintaining air-core photonic bandgap fiber (PM-PBF). The experimental results show that the Verdet constant of the PM-PBF is $\sim 3.3 \mathrm{mrad} / \mathrm{T} / \mathrm{m}$ for the broadband light with a spectral width of $\sim 38 \mathrm{~nm}$ and a mean wavelength of $\sim 1550 \mathrm{~nm}$, which is $\sim 124$ times less than that of a conventional stress-induced birefringent fibers called PANDA fibers ( $\sim 0.41 \mathrm{rad} / \mathrm{T} / \mathrm{m}$ for the same broad-spectrum light). The results indicate that the nonreciprocal error induced by the Faraday effect in a fiber optic gyroscope (FOG) made of the PM-PBF is theoretically $\sim 25$ times less than that of a conventional FOG made of the PANDA fiber when other conditions, such as the fiber twist, fiber coil area, and so on, are the same.
\end{abstract}

Keywords: Faraday effect; gyroscopes; microstructured fibers

\section{Introduction}

The Faraday effect may deteriorate the performance of a fiber optic gyroscope (FOG) when the sensing coil of the FOG is exposed to a geomagnetic field [1,2]. Protecting the coil with a magnetic shield is the strategy usually adopted to reduce the impact of the Faraday effect on the FOG performance. The magnetic shield, however, introduces additional weight, cost, and complexity, and, as a result, limits the FOG's applications.

Photonic crystal fiber (PCF) is a class of optical fiber based on two-dimensional photonic crystal, and strongly attracts researchers' interest owing to its special characteristics. New PCFs emerge constantly according to different applications, such as the recent composite PCFs [3,4], nodeless air-core PCFs [5], and so on. Among those fibers, air-core photonic bandgap fiber (PBF) offers a radically new method for solving the problem of environment (including magnetic field) adaptation, because the PBF causes light to propagate in air that exhibits much lower sensitivity to magnetic fields than conventional $\mathrm{SiO}_{2}$ [6]. Thus, a polarization-maintaining air-core $\mathrm{PBF}$ (PM-PBF) is expected to be more effective in reducing the Faraday effect in a FOG [7].

A. M. Smith $[8,9]$ measured the Verdet constant of a single-mode optical fiber, and found it to be $\sim 3.61 \mathrm{rad} / \mathrm{T} / \mathrm{m}$ at $632.8 \mathrm{~nm}$ and $\sim 2.05 \mathrm{rad} / \mathrm{T} / \mathrm{m}$ at $830 \mathrm{~nm}$. J. L. Cruz et al. [10] measured the effective Verdet constant of a standard single-mode fiber and found it to be $0.54 \pm 0.02 \mathrm{rad} / \mathrm{T} / \mathrm{m}$ at $1523 \mathrm{~nm}$. J. Noda et al. [11] measured the Verdet constant of a conventional PANDA fiber at different wavelengths, showing it to be $\sim 0.60 \mathrm{rad} / \mathrm{T} / \mathrm{m}$ at $1550 \mathrm{~nm}$. H. Wen et al. [12] reported the first measurement of the Verdet constant of a seven-cell non-PM PBF, $6.1 \pm 0.3 \mathrm{mrad} / \mathrm{T} / \mathrm{m}$ at $1545 \mathrm{~nm}$, which is about 90 times less than that of standard single-mode fiber (Corning's SMF-28). L. Sun et al. $[13,14]$ measured the effective Verdet constant of a $25 \mathrm{wt}$. \% terbium-doped-core phosphate 
fiber and a $56 \mathrm{wt}$. \% terbium (Tb)-doped silicate fiber, which were found to be $-6.2 \pm 0.4 \mathrm{rad} / \mathrm{T} / \mathrm{m}$ and $-24.5 \pm 1.0 \mathrm{rad} / \mathrm{T} / \mathrm{m}$ at $1053 \mathrm{~nm}$, respectively. Although the Verdet constants of many kinds of fibers have been reported, the Verdet constant of a PM-PBF remains unreported. What is more, the light source used in previous studies is a laser with a narrow spectral width, and the obtained Verdet constant is only credible at a certain wavelength, which is not suitable for an accurate analysis of the nonreciprocal error induced by the Faraday effect in a FOG, because there is always a broadband light source with a spectral width of tens of nanometers employed in a FOG.

Therefore, in this paper, we report the measurement of the Verdet constant of a PM-PBF using the white-light interference technique based on a broad-spectrum amplified spontaneous emission (ASE) source and an all-fiber setup. This result can provide a foundation for the analysis of both the magnetic properties in a PM-PBF and nonreciprocal error induced by the Faraday effect in a polarization-maintaining air-core photonic-bandgap FOG (PM-PBFOG).

\section{Measurement Principle}

The schematic of the Verdet constant measuring setup is shown in Figure 1, which is based on the configuration of Reference [11]. Light from an ASE source is launched into integrated optic chip (IOC) A. The IOC has an extinction ratio of more than $70 \mathrm{~dB}$, so the output light becomes a linear polarization beam $W_{p}$. Next, $W_{p}$ is coupled into the PM-PBF $(\sim 60 \mathrm{~cm})$ under test. The PM-PBF passes through a $\sim 1 \mathrm{~mm}$-diameter bore in the center of an electromagnet, and the width of the pole-gap of the electromagnet is $\mathrm{L} \approx 1 \mathrm{~mm}$. The electromagnet is placed near the fusion splicing point $\mathrm{P}_{2}$ and excited by a sinusoidal electrical signal, generated by a power amplifier connected to a signal generator. Meanwhile, the PM-PBF is fixed on a one-dimensional motorized translation stage to realize precise movements in the magnetic field. Finally, the light is converted to an electronic signal by the detector after passing through IOC B. A lock-in amplifier is used to demodulate this electronic signal and resolve the Verdet constant of the PM-PBF.

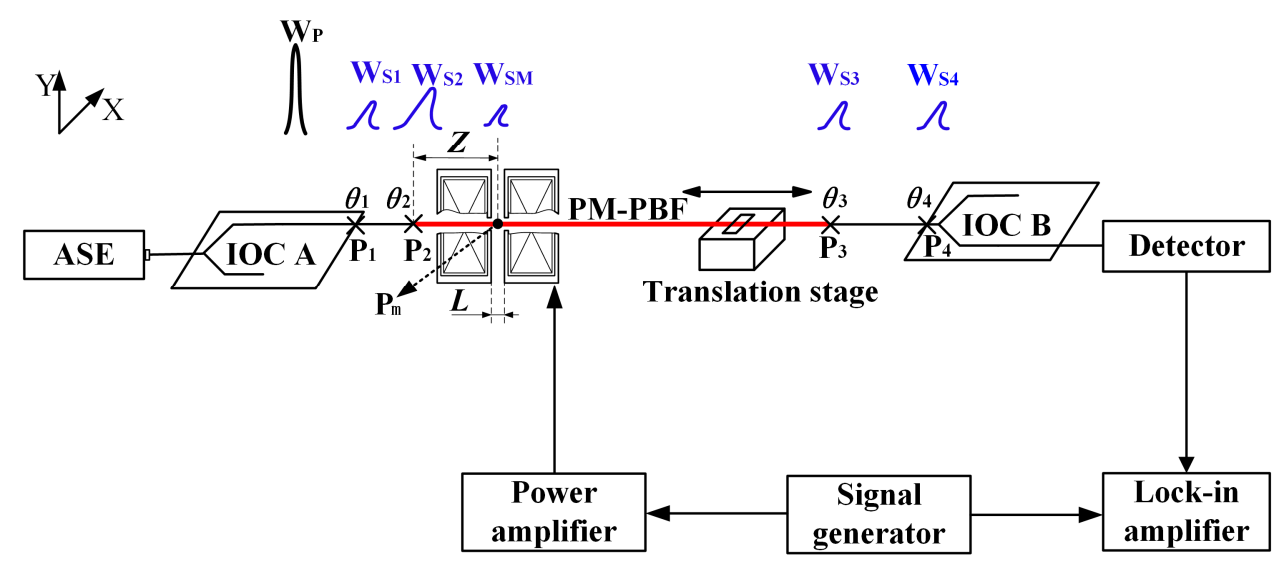

Figure 1. Schematic of the setup for measuring the Verdet constant of the polarization-maintaining air-core photonic bandgap fiber (PM-PBF). The bold red line between $\mathrm{P}_{2}$ and $\mathrm{P}_{3}$ is the PM-PBF.

In Figure $1, \mathrm{P}_{1}, \mathrm{P}_{2}, \mathrm{P}_{3}$, and $\mathrm{P}_{4}$ are the coupling or fusion splicing points between IOC $\mathrm{A}$ and its pigtail, IOC A's pigtail and the PM-PBF, the PM-PBF and IOC B's pigtail, and IOC B's pigtail and IOC B, respectively. The alignment angles between birefringent axes of the related two parts at the points $P_{1}$, $P_{2}, P_{3}$, and $P_{4}$ are assumed to be $\theta_{1}, \theta_{2}, \theta_{3}$, and $\theta_{4}$, respectively. $\theta_{1}, \theta_{2}$, and $\theta_{4}$ are around $0^{\circ} ; \theta_{3}$ is about $90^{\circ}$. When the polarized primary wave $\mathrm{W}_{\mathrm{P}}$ propagates through $\mathrm{P}_{1}, \mathrm{P}_{2}, \mathrm{P}_{3}$, and $\mathrm{P}_{4}$, the polarization crossover-induced secondary waves $W_{S 1}, W_{S 2}, W_{S 3}$, and $W_{S 4}$ are produced, with a polarization state perpendicular to that of $W_{P}$, because the cross-coupling exists at those coupling or fusion splicing points. Meanwhile, an equivalent rotation angle $\theta_{\mathrm{m}}$ is caused by the Faraday effect at the point $\mathrm{P}_{\mathrm{m}}$ within the electromagnet, so another secondary wave $W_{\mathrm{SM}}$ also arises when $W_{\mathrm{p}}$ passes through the 
point $P_{m}$ [11]. Considering the polarization states of the primary and secondary waves, we find that the primary wave $\mathrm{W}_{\mathrm{P}}$ is eliminated by the polarizer within IOC $\mathrm{B}$, and only $\mathrm{W}_{\mathrm{S} 1}, \mathrm{~W}_{\mathrm{S} 2}, \mathrm{~W}_{\mathrm{S} 3}, \mathrm{~W}_{\mathrm{S} 4}$, and $W_{S M}$ are able to arrive at the detector; their optical paths are shown in Table 1 . The amplitude of $\mathrm{W}_{\mathrm{SM}}$ reflects the Verdet constant of the PM-PBF, so the purpose of this work is to resolve the amplitude of $W_{S M}$.

Table 1. Optical path of secondary waves.

\begin{tabular}{cc}
\hline Secondary Waves & Optical Path \\
\hline$W_{\mathrm{S} 1}$ & $\mathrm{n}_{\mathrm{s} 1} L_{\mathrm{P} 1-\mathrm{P} 2}+\mathrm{n}_{\mathrm{s} 2}\left(\mathrm{Z}+\mathrm{L}_{\mathrm{Pm}-\mathrm{P} 3}\right)+\mathrm{n}_{\mathrm{f} 3} L_{\mathrm{P} 3-\mathrm{P} 4}$ \\
$W_{\mathrm{S} 2}$ & $\mathrm{n}_{\mathrm{f} 1} L_{\mathrm{P} 1-\mathrm{P} 2}+\mathrm{n}_{\mathrm{s} 2}\left(\mathrm{Z}+\mathrm{L}_{\mathrm{Pm}-\mathrm{P} 3}\right)+\mathrm{n}_{\mathrm{f} 3} L_{\mathrm{P} 3-\mathrm{P} 4}$ \\
$W_{\mathrm{S} M}$ & $\mathrm{n}_{\mathrm{f} 1} L_{\mathrm{P} 1-\mathrm{P} 2}+\mathrm{n}_{\mathrm{f} 2} \mathrm{Z}+\mathrm{n}_{\mathrm{s} 2} L_{\mathrm{Pm}-\mathrm{P} 3}+\mathrm{n}_{\mathrm{f} 3} L_{\mathrm{P} 3-\mathrm{P} 4}$ \\
$W_{\mathrm{S} 3}$ & $\mathrm{n}_{\mathrm{f} 1} L_{\mathrm{P} 1-\mathrm{P} 2}+\mathrm{n}_{\mathrm{f} 2}\left(\mathrm{Z}+\mathrm{L}_{\mathrm{Pm}-\mathrm{P} 3}\right)+\mathrm{n}_{\mathrm{f} 3} L_{\mathrm{P} 3-\mathrm{P} 4}$ \\
$W_{\mathrm{S} 4}$ & $\mathrm{n}_{\mathrm{f} 1} L_{\mathrm{P} 1-\mathrm{P} 2}+\mathrm{n}_{\mathrm{f} 2}\left(\mathrm{Z}+\mathrm{L}_{\mathrm{Pm}-\mathrm{P} 3}\right)+\mathrm{n}_{\mathrm{s} 3} L_{\mathrm{P} 3-\mathrm{P} 4}$ \\
\hline
\end{tabular}

$\mathrm{n}_{\mathrm{s} 1}$ and $\mathrm{n}_{\mathrm{f} 1}, \mathrm{n}_{\mathrm{s} 2}$ and $\mathrm{n}_{\mathrm{f} 2}, \mathrm{n}_{\mathrm{s} 3}$ and $\mathrm{n}_{\mathrm{f} 3}$ denote the refractive index of the slow and fast axes of IOC A's pigtail, $\mathrm{PM}-\mathrm{PBF}$, and IOC B's pigtail, respectively. $\mathrm{L}_{\mathrm{P} 1-\mathrm{P} 2}, \mathrm{Z}, \mathrm{L}_{\mathrm{Pm}-\mathrm{P} 3}$, and $\mathrm{L}_{\mathrm{P} 3-\mathrm{P} 4}$ denote the distance between $\mathrm{P}_{1}$ and $\mathrm{P}_{2}, \mathrm{P}_{2}$ and $\mathrm{P}_{\mathrm{m}}, \mathrm{P}_{\mathrm{m}}$ and $\mathrm{P}_{3}$, as well as $\mathrm{P}_{3}$ and $\mathrm{P}_{4}$, respectively.

If the source is the laser that was used in References [8-14], all of these wavetrains $\left(\mathrm{W}_{\mathrm{S} 1}, \mathrm{~W}_{\mathrm{S} 2}\right.$, $\mathrm{W}_{\mathrm{S} 3}, \mathrm{~W}_{\mathrm{S} 4}$, and $\left.\mathrm{W}_{\mathrm{SM}}\right)$ at the detector would interfere and the situation would be very complicated. However, as an ASE source with a very short coherence length $(\sim 61.9 \mu \mathrm{m})$ is used here, we can make the optical path difference sufficiently small between $W_{\mathrm{SM}}$ and $W_{\mathrm{S} 2}$ (see Table 1 ), which means that the interference only occurs between $\mathrm{W}_{\mathrm{SM}}$ and $\mathrm{W}_{\mathrm{S} 2}$, by optimizing the location of the electromagnet and lengths of the PM-PBF, IOC A's pigtail, and IOC B's pigtail. As a result, $\mathrm{W}_{\mathrm{S} 2}$ actually becomes a reference wavetrain to interfere with the signal wavetrain $W_{S M}$. Then, the situation becomes very simple, and the interference intensity between $\mathrm{W}_{\mathrm{S} 2}$ and $\mathrm{W}_{\mathrm{SM}}$ at the detector is given by:

$$
V_{\text {interference }}=V_{\mathrm{S} 2}+V_{\mathrm{SM}}+2 \sqrt{V_{\mathrm{S} 2} V_{\mathrm{SM}}} \gamma\left(\frac{\Delta \phi \lambda}{2 \pi c}\right) \cos \Delta \phi
$$

where $V_{\mathrm{S} 2}$ and $V_{\mathrm{SM}}$ are voltages corresponding to $\mathrm{W}_{\mathrm{S} 2}$ and $\mathrm{W}_{\mathrm{SM}}$ at the detector, respectively, and they are given by the first two formulas in Equation (2). In addition, $\gamma$ is the coherence function of the ASE source [7], $\Delta \phi$ is the phase difference between $\mathrm{W}_{\mathrm{S} 2}$ and $\mathrm{W}_{\mathrm{SM}}$ (given by the third formula in Equation (2)), $\lambda$ is the mean wavelength of the source, and $c$ is the velocity of light.

$$
\left\{\begin{array}{l}
V_{\mathrm{S} 2}=\left(P_{0} R / \alpha_{1} \alpha_{2}\right) \cos ^{2} \theta_{1} \cdot \sin ^{2} \theta_{2} \\
V_{\mathrm{SM}}=\left(P_{0} R / \alpha_{1} \alpha_{2}\right) \cos ^{2} \theta_{1} \cdot \cos ^{2} \theta_{2} \cdot \sin ^{2} \theta_{\mathrm{m}} \\
\Delta \phi=2 \pi \mathrm{Z} / L_{\mathrm{B}}
\end{array}\right.
$$

In Equation (2), $P_{0}$ is power of the ASE source, $R$ is the conversion efficiency of the detector, $\alpha_{1}$ denotes the loss from the ASE source to the point $P_{m}, \alpha_{2}$ denotes the loss from the point $P_{m}$ to the detector, and $Z$ is the distance between the points $\mathrm{P}_{2}$ and $\mathrm{P}_{\mathrm{m}} . \theta_{\mathrm{m}}$ caused by the Faraday effect is $V B \sin$ $(\omega t) L \sin (\delta / 2) /(\delta / 2)$ [11], where $V$ is Verdet constant of the fiber, $B$ is intensity of the magnetic field and is approximately constant within the pole gap, $\omega$ is modulation frequency of the magnetic field applied to the PM-PBF, $\delta=2 \pi L / L_{\mathrm{B}}$, and $L_{\mathrm{B}}$ is beat length of the fiber.

The extremely low magnetic-field sensitivity of the PM-PBF causes $\theta_{\mathrm{m}}$ and $V_{\mathrm{SM}}$ to be extremely small, so it is impossible for the direct detection of the interference signal $V_{\text {interference. Thus, coherence }}$ detection must be applied here. A sinusoidal magnetic field is applied to the PM-PBF at the point $P_{m}$ to modulate $V_{\mathrm{SM}}$ and the interference signal ( $\left.V_{\text {interference }}\right)$, and only the third term of Equation (1) can 
be resolved because only this term has the same frequency component with the modulation frequency $\omega$ [15]. Then, the demodulation signal ( $\left.V_{\text {demodulation }}\right)$ resolved by the lock-in amplifier is given by:

$$
V_{\text {demodulation }} \approx \frac{P_{0} R V B L_{\mathrm{B}} \gamma\left(\frac{Z \lambda}{L_{\mathrm{B} C}}\right) \sin \left(\frac{\pi L}{L_{\mathrm{B}}}\right) \cdot \cos ^{2} \theta_{1} \cdot \sin 2 \theta_{2} \cdot \cos \left(\frac{2 \pi Z}{L_{\mathrm{B}}}\right)}{\sqrt{2} \pi \alpha_{1} \alpha_{2}}
$$

Thus, $V_{\text {demodulation }}$ demonstrates an approximately sinusoidal oscillation as $Z$ changes linearly. Furthermore, its maximum peak-to-peak value $\left(V_{\text {demodulation-p-to-p }}\right)$ reveals the Verdet constant $(V)$ of the PM-PBF to be:

$$
V \approx \frac{\pi \alpha_{1} \alpha_{2} V_{\text {demodulation }-\mathrm{p}-\mathrm{to}-\mathrm{p}}}{\sqrt{2} P_{0} R B L_{\mathrm{B}} \sin \left(\frac{\pi L}{L_{\mathrm{B}}}\right) \cdot \cos ^{2} \theta_{1} \cdot \sin 2 \theta_{2}}
$$

Compared with the other methods in previous studies used to determine the Verdet constant [8-14], not a laser source but a broad-spectrum ASE source and the white-light interference technique is used here, so the measured Verdet constant is more accurate and can be applied to the analysis of the nonreciprocal error induced by the Faraday effect in a FOG. Meanwhile, the ASE source has a very short coherence length, so it can avoid unwanted interference between irrelevant secondary waves, which is very important to simplify the analysis and improve the measurement system performance. Moreover, all optical components of the measuring setup employ all-fiber connections, which can avoid secondary waves induced by interface reflection and improve the system reliability and stability.

\section{Experimental Results}

An experiment setup based on Figure 1 is established to measure the Verdet constant of a commercial seven-cell PM-PBF (see the left inset in Figure 2a) with an air filling ratio of $\sim 97 \%$, a loss of $\sim 25 \mathrm{~dB} / \mathrm{km}$, a mode diameter of $\sim 9 \mathrm{um}$, and a cladding diameter of $\sim 120 \mathrm{um}$, respectively. The ASE source has a flat spectrum with a width of $\sim 38 \mathrm{~nm}$ and a mean wavelength of $\sim 1550 \mathrm{~nm}$. The detector's conversion efficiency $\mathrm{R}$ is $\sim 0.038 \mathrm{~V} / \mu \mathrm{W}$. The fusion splicing angle $\theta_{1} \approx 1^{\circ}$, and $\theta_{2}$ is deliberately made larger $\left(\sim 6^{\circ}\right)$ to increase the intensity of the reference wavetrain $W_{\mathrm{S} 2}$ but avoid saturation of the detector at the same time. The magnetic-field intensity $B$ is modulated by a $30-\mathrm{Hz}$ sinusoidal signal, and has an amplitude of $\sim 0.25 \mathrm{~T}$ as a result.

When the PM-PBF is precisely moved forward by the translation stage to make the magnetic position $\left(\mathrm{P}_{\mathrm{m}}\right)$ uniformly scan from the right to the left of the fusion splicing point $\mathrm{P}_{2}$ (see Figure 1 ), the demodulation value ( $\left.V_{\text {demodulation }}\right)$ of the interference signal $\left(V_{\text {interference }}\right)$ is presented in Figure $2 \mathrm{a}$. The demodulation value within the dashed- and solid-line rectangle in Figure 2a corresponds to the PM-PBF (see the left inset) and conventional PANDA fiber (see the right inset) that has a loss of $\sim 0.27 \mathrm{~dB} / \mathrm{km}$, a mode diameter of $\sim 6.5 \mathrm{um}$, and a cladding diameter of $\sim 125 \mathrm{um}$, respectively. Furthermore, it is interesting to note that there is a transition area in the middle which seems to be a little disorganized. This is reasonable because the states of the fibers, especially the air holes in the PM-PBF, have changed in the process of fusion splicing. Therefore, the experimental results within this area are not accurate for the analysis of the Verdet constant of the PM-PBF and conventional PANDA fibers, and we utilize the data slightly farther from this area. For the conventional PANDA fiber, as illustrated in the solid-line rectangle in Figure $2 \mathrm{a}, V_{\text {demodulation }}$ sinusoidally varies with the position $\mathrm{P}_{\mathrm{m}}$ of the magnetic field imposed on the fiber, which agrees well with the theoretical expectation in the previous section. It shows that the oscillation period is $\sim 2.6 \mathrm{~mm}$, which actually means that the beat length $L_{\mathrm{B}}$ equals $\sim 2.6 \mathrm{~mm}$ [16]. The peak-to-peak value of the demodulation signal is $V_{\text {demodulation-P-to-P-1 }}=\sim 1.42 \mathrm{mV}$. Therefore, the Verdet constant of the conventional PANDA fiber is $\sim 0.41 \mathrm{rad} / \mathrm{T} / \mathrm{m}$ based on Equation (4). 

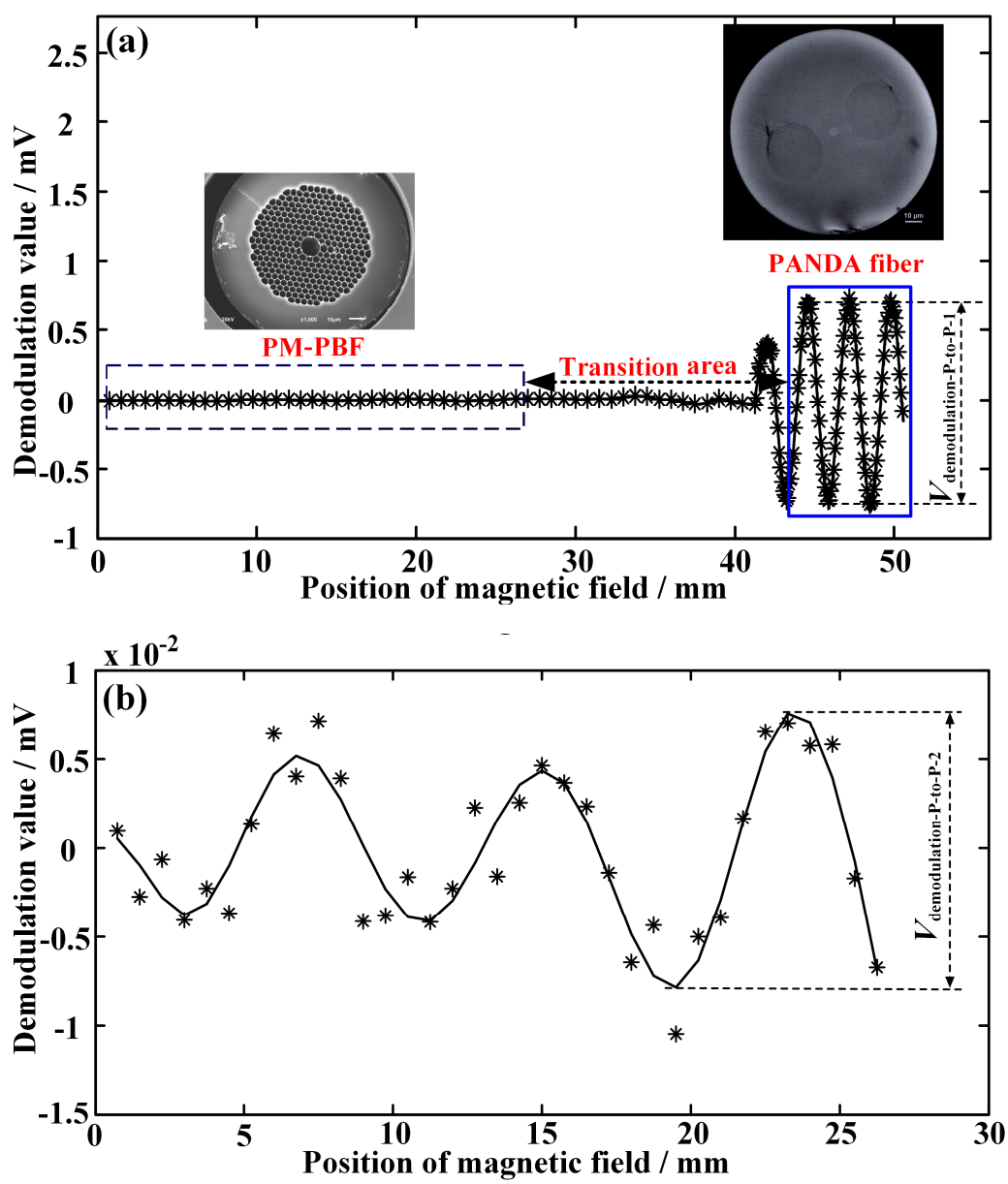

Figure 2. Test results of the Verdet constants of the PM-PBF and conventional PANDA fiber. (a) $V_{\text {demodulation }}$ sinusoidally oscillates as the position $\left(\mathrm{P}_{\mathrm{m}}\right)$ of the magnetic field uniformly scans from the right to the left of the fusion splicing point $P_{2}$ in Figure 1. (b) Enlarged image of the area within the dashed-line rectangle in (a).

For the PM-PBF, the enlarged image of the corresponding demodulated signal within the dashed-line rectangle in Figure 2a is shown in Figure $2 b$. Obviously, the oscillation period is $\sim 7.75 \mathrm{~mm}$, which means that the beat length of the PM-PBF is $\sim 7.75 \mathrm{~mm}$ [16]. The peak-to-peak value of the demodulation signal is $V_{\text {demodulation-P-to-P-2 }}=\sim 17.5 \mu \mathrm{V}$. Consequently, the Verdet constant of the PM-PBF equals $\sim 3.3 \mathrm{mrad} / \mathrm{T} / \mathrm{m}$, which is $\sim 124$ times less than that of the conventional PANDA fiber.

The experiment is performed at room temperature, and the most sensitive component to temperature is the birefringence of the polarization-maintaining fiber. However, the temperaturevariation-induced error of the Verdet constant can be ignored because it is a few orders of magnitude smaller than the absolute value. According to the theoretical analysis in Reference [2], the nonreciprocal error induced by the Faraday effect in a FOG is proportional to the ratio of the Verdet constant $(V)$ to the birefringence $(\Delta \beta)$. Therefore, based on our measurement results of the Verdet constant and beat length, the nonreciprocal error induced by the Faraday effect in a PM-PBFOG is theoretically 25 times less than that of a conventional FOG made of PANDA fiber coil when the other conditions, such as the fiber twist, fiber coil area, and so on, are the same. Similarly, this error in PM-PBFOG is theoretically $\sim 19$ times less than that of a FOG made of a seven-cell non-PM PBF [12].

\section{Conclusions}

In summary, we proposed a method based on the white-light interference technique to measure the Verdet constant of a PM-PBF. The setup employs an ASE source with a spectral width of $\sim 38 \mathrm{~nm}$ 
and a mean wavelength of $\sim 1550 \mathrm{~nm}$. The results show that the Verdet constants of the PM-PBF and the conventional PANDA fiber are, respectively, $\sim 3.3 \mathrm{mrad} / \mathrm{T} / \mathrm{m}$ and $\sim 0.41 \mathrm{rad} / \mathrm{T} / \mathrm{m}$, indicating that the nonreciprocal error induced by the Faraday effect in a FOG made of the PM-PBF is theoretically $\sim 25$ times less than that of a conventional FOG made of the PANDA fiber when the other conditions, such as the fiber twist, fiber coil area, and so on, are the same. Therefore, the experimental results provide an important foundation for the analysis of the magnetic properties of a PM-PBF and the Faraday effect in a PM-PBFOG.

Acknowledgments: This work was supported by National Natural Science Foundation of China (NSFC) under grants No. 61575012 and 61575013, National Key Scientific Instrument and Equipment Development Project of China under grant No. 2013YQ040877.

Author Contributions: Ningfang Song and Xiaobin $\mathrm{Xu}$ promoted the experimental methods and setup; Xiaoyang Wang performed the experiments and wrote the paper; Xiaoyang Wang and Cai Wei analyzed the data; Chunxiao $\mathrm{Wu}$ fused the fusion splicing points.

Conflicts of Interest: The authors declare no conflict of interest.

\section{References}

1. Böhm, K.; Petermann, K.; Weidel, E. Sensitivity of a fiber-optic gyroscope to environmental magnetic fields. Opt. Lett. 1982, 7, 180-182. [CrossRef] [PubMed]

2. Hotate, K.; Tabe, K. Drift of an optical fiber gyroscope caused by the Faraday effect: Influence of the earth's magnetic field. Appl. Opt. 1986, 25, 1086-1092. [CrossRef] [PubMed]

3. Markos, C.; Yannopoulos, S.N.; Vlachos, K. Chalcogenide glass layers in silica photonic crystal fibers. Opt. Express 2012, 20, 14814-14824. [CrossRef] [PubMed]

4. Konidakis, I.; Zito, G.; Pissadakis, S. Silver plasmon resonance effects in $\mathrm{AgPO}_{3} /$ silica photonic bandgap fiber. Opt. Lett. 2014, 39, 3374-3377. [CrossRef] [PubMed]

5. Gao, S.F.; Wang, Y.Y.; Liu, X.L.; Hong, C.; Gu, S.; Wang, P. Nodeless hollow-core fiber for the visible spectral range. Opt. Lett. 2017, 42, 61. [CrossRef] [PubMed]

6. Kim, H.K.; Digonnet, M.J.F.; Kino, G.S. Air-core photonic-bandgap fiber-optic gyroscope. J. Lightwave Technol. 2006, 24, 3169-3174.

7. Lefèvre, H.C. The Fiber-Optic Gyroscope, 2nd ed.; Artech House Press: Boston, MA, USA, 2014.

8. Smith, A.M. Polarization; magnetooptic properties of single-mode optical fiber. Appl. Opt. 1978, 17, 52-56. [CrossRef] [PubMed]

9. Smith, A.M. Faraday effect in single-mode optical fibre using an injection laser light source. Electron. Lett. 1980, 16, 206-208. [CrossRef]

10. Cruz, J.L.; Andres, M.V.; Hernandez, M.A. Faraday effect in standard optical fibers: Dispersion of the effective Verdet constant. Appl. Opt. 1996, 35, 922-927. [CrossRef] [PubMed]

11. Noda, J.; Hosaka, T.; Sasaki, Y.; Ulrich, R. Dispersion of Verdet constant in stress-birefringent silica fibre. Electron. Lett. 1984, 20, 906-908. [CrossRef]

12. Wen, H.; Terrel, M.A.; Kim, H.K.; Digonnet, M.J.; Fan, S. Measurements of the Birefringence and Verdet Constant in an Air-Core Fiber. J. Lightwave Technol. 2009, 27, 3194-3201. [CrossRef]

13. Sun, L.; Jiang, S.; Zuegel, J.D.; Marciante, J.R. Effective verdet constant in a terbium-doped-core phosphate fiber. Opt. Lett. 2009, 34, 1699-1701. [CrossRef] [PubMed]

14. Sun, L.; Jiang, S.; Zuegel, J.D.; Marciante, J.R. All-fiber optical isolator based on faraday rotation in highly terbium-doped fiber. Opt. Lett. 2010, 35, 706-708. [CrossRef] [PubMed]

15. Gao, J. Detection of Weak Signals; Tsinghua University Press: Beijing, China, 2004.

16. Zhang, P.G.; Halliday, D.I. Measurement of the beat length in high-birefringent optical fiber by way of magnetooptic modulation. J. Lightwave Technol. 1994, 12, 597-602. [CrossRef]

(C) 2017 by the authors. Licensee MDPI, Basel, Switzerland. This article is an open access article distributed under the terms and conditions of the Creative Commons Attribution (CC BY) license (http://creativecommons.org/licenses/by/4.0/). 